\title{
Endogenous, cholesterol-activated ATP-dependent transport in membrane vesicles from Spodoptera frugiperda cells
}

\section{Sjöstedt, Noora}

2019-09-01

Sjöstedt , N , Salminen , T A \& Kidron , H 2019 , ' Endogenous, cholesterol-activated ATP-dependent transport in membrane vesicles from Spodoptera frugiperda cells ' , European Journal of Pharmaceutical Sciences , vol. 137 , 104963 . https://doi.org/10.1016/j.ejps.2019.104963

http://hdl.handle.net/10138/316256

https://doi.org/10.1016/j.ejps.2019.104963

cc_by_nc_nd

acceptedVersion

Downloaded from Helda, University of Helsinki institutional repository.

This is an electronic reprint of the original article.

This reprint may differ from the original in pagination and typographic detail.

Please cite the original version. 


\section{Accepted Manuscript}

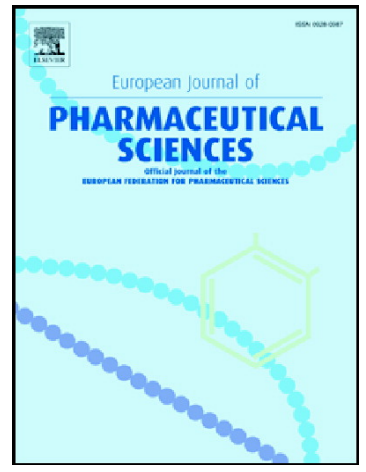

Noora Sjöstedt, Tiina A. Salminen, Heidi Kidron

PII: $\quad$ S0928-0987(19)30226-X

DOI: $\quad$ https://doi.org/10.1016/j.ejps.2019.104963

Article Number: $\quad 104963$

Reference: $\quad$ PHASCI 104963

To appear in: $\quad$ European Journal of Pharmaceutical Sciences

Received date: $\quad 10$ December 2018

Revised date: $\quad 4$ June 2019

Accepted date: $\quad 17$ June 2019

Please cite this article as: N. Sjöstedt, T.A. Salminen and H. Kidron, Endogenous, cholesterol-activated ATP-dependent transport in membrane vesicles from Spodoptera frugiperda cells, European Journal of Pharmaceutical Sciences, https://doi.org/10.1016/ j.ejps.2019.104963

This is a PDF file of an unedited manuscript that has been accepted for publication. As a service to our customers we are providing this early version of the manuscript. The manuscript will undergo copyediting, typesetting, and review of the resulting proof before it is published in its final form. Please note that during the production process errors may be discovered which could affect the content, and all legal disclaimers that apply to the journal pertain. 
Endogenous, cholesterol-activated ATP-dependent transport in membrane vesicles from Spodoptera frugiperda cells

Noora Sjöstedt ${ }^{1^{*}}$, Tiina A. Salminen ${ }^{2}$ and Heidi Kidron ${ }^{1}$

${ }^{1}$ Drug Research Program, Division of Pharmaceutical Biosciences, Faculty of Pharmacy, University of Helsinki, Helsinki, Finland

${ }^{2}$ Structural Bioinformatics Laboratory, Biochemistry, Faculty of Science and Engineering, Åbo Akademi University, Turku, Finland

* Corresponding author, e-mail noora.sjostedt@helsinki.fi, tel. +1 (919) 6991140 


\section{Abstract}

Transport proteins of the ATP-binding cassette (ABC) family are found in all kingdoms of life. In humans, several $A B C$ efflux transporters play a role in drug disposition and excretion. Therefore, in vitro methods have been developed to characterize the substrate and inhibitor properties of drugs with respect to these transporters. In the vesicular transport assay, transport is studied using inverted membrane vesicles produced from transporter overexpressing cell lines of both mammalian and insect origin. Insect cell expression systems benefit from a higher expression compared to background, but are not as well characterized as their mammalian counterparts regarding endogenous transport. Therefore, the contribution of this transport in the assay might be underappreciated. In this study, endogenous transport in membrane vesicles from Spodoptera frugiperda -derived Sf9 cells was characterized using four typical substrates of human $A B C$ transporters: 5(6)-carboxy-2, $7^{\prime}$-dichlorofluorescein (CDCF), estradiol-17 $\beta$ glucuronide, estrone sulfate and N-methyl-quinidine. Significant ATP-dependent transport was observed for three of the substrates with cholesterol-loading of the vesicles, which is sometimes used to improve the activity of human transporters expressed in Sf9 cells. The highest effect of cholesterol was on CDCF transport, and this transport in the cholesterol-loaded Sf9 vesicles was time and concentration dependent with a $\mathrm{K}_{\mathrm{m}}$ of $8.06 \pm 1.11 \mu \mathrm{M}$. The observed CDCF transport was inhibited by known inhibitors of human $A B C C$ transporters, but not by $A B C B 1$ and $A B C G 2$ inhibitors verapamil and Ko143, respectively. Two candidate genes for ABCC-type transporters in the S. frugiperda genome (SfABCC2 and SfABCC3) were identified based on sequence analysis as a hypothesis to explain the observed endogenous ABCC-type transport in Sf9 vesicles. Although further studies are needed to verify the role of SfABCC2 and SfABCC3 in Sf9 vesicles, the findings of this study highlight the need to carefully characterize background transport in Sf9 derived membrane vesicles to avoid false positive substrate findings for human $A B C$ transporters studied with this overexpression system.

\section{Key words}

Sf9, $A B C$ transporter, $C D C F$, estradiol glucuronide, estrone sulfate, $\mathrm{N}$-methyl quinidine

\section{Introduction}

The ATP-binding cassette $(A B C)$ protein family is one of the largest transporter families and it can be found in all kingdoms of life. The family can be divided into several subfamilies (e.g. seven subfamilies, named A$G$ in humans)(Dean et al., 2001). A functional $A B C$ transporter typically contains four domains; two intracellular nucleotide binding domains (NBD) and two transmembrane domains (TMD). The hydrolysis of 
ATP bound to the NBD, provides the energy required to transport substrates over cellular membranes. In eukaryotes, most members of the $A B C$ family work as efflux transporters to pump a diverse range of both endogenous and exogenous compounds from the cytoplasm to the outside of cells or into organelles, while prokaryotes also express $A B C$ transporters that mediate the uptake of substrates (Higgins, 1992).

In humans, $A B C$ transporters are expressed at physiological barrier tissues, where they can affect the absorption, distribution and elimination of endogenous compounds and substrate drugs (Giacomini et al., 2010). Some of these transporters are also overexpressed in tumour tissues, where they can cause drug resistance (Gottesman et al., 2002). Not all $A B C$ transporters have drug substrates, but at least $A B C B 1$ (also called P-glycoprotein or multi-drug resistance protein 1, MDR1), ABCC 2-4 (multi-drug resistance associated protein 2-4, MRP2-4) and ABCG2 (breast cancer resistance protein, BCRP) are known to influence drug disposition and may have clinically significant consequences (Giacomini et al., 2010; Hillgren et al., 2013). Therefore, it is of interest to study the transport activity and inhibition of $A B C$ transporters in drug development.

Transport can be studied in a variety of experimental systems, including intact cells and inverted plasma membrane vesicles that are spontaneously formed after cell lysis (Brouwer et al., 2013). Membrane vesicles from transporter overexpressing cells may facilitate studies to identify transport via specific transporters or transporter inhibition. Vesicle studies are especially beneficial for hydrophilic efflux substrates that rely on active mechanisms to enter cells or metabolic products that are formed within cells. Membrane vesicles can be formed from any cells, but continuous cell lines from Spodoptera frugiperda ovaries (Sf9, Sf21) have been frequently used for baculovirus transfection and the overexpression of human transporters (Glavinas et al., 2004). The advantages of the insect cells compared to mammalian or human cell lines are the lack of background expression of the studied human/mammalian transporters, ease of culture, higher throughput and cost-efficiency. Nowadays, however, many transporters are expressed and commercially available in mammalian cell derived membrane vesicles as well as insect cell derived.

Despite the benefits of the insect expression systems, they have some properties that could affect the activity of expressed human transporters. One of the important factors is the difference between insect and mammalian cells in membrane composition. $A B C$ transporters tend to localize in cholesterol enriched areas, or lipid rafts, in membranes (Guyot and Stieger, 2011; Meyer dos Santos et al., 2007; Storch et al., 2007) and, therefore, the lipid environment, especially cholesterol content, is known to affect the function of certain transporters (Eckford and Sharom, 2008; Fenyvesi et al., 2008; Guyot et al., 2014; Pal et al., 2007; Telbisz et al., 2007). The molar cholesterol:phospholipid ratio in Sf9 cells is approximately 10- to 20-fold lower than in higher eukaryotic cells (Gimpl et al., 1995; Marheineke et al., 1998) and the cholesterol concentration in Sf9 vesicles is reported to be 4 - to 5 -fold lower than in vesicles produced from a 
mammalian cell line (Pal et al., 2007). To compensate for the lower cholesterol content in Sf9 cells, cholesterol is often loaded to the membrane vesicles in order to improve the activity of transporters that are sensitive to cholesterol. This artificial cholesterol loading increases the transport activity of the expressed transporters (Guyot et al., 2014; Pal et al., 2007; Telbisz et al., 2007).

Endogenous transport activity has been reported in many human and mammalian cell lines that are used to express and study specific transporters during drug development (Ahlin et al., 2009; Brouwer et al., 2013; Goh et al., 2002). This endogenous transport could be a confounding factor in membrane vesicle assays if not accounted for by control measurements from non-transfected vesicles (Brouwer et al., 2013). Although the insect cell expression systems benefit from a higher expression compared to background than mammalian cell lines (Glavinas et al., 2008), Sf9 and Sf21 cells express endogenous drug transporters of $S$. frugiperda. However, these insect transporters are not as well characterized as their mammalian counterparts and their contribution to apparent transport in the assay might be underappreciated. In this article, we present the endogenous ATP-dependent transport in membrane vesicles produced from Sf9 cells that is activated by cholesterol-loading of the membranes. Based on studies with several probe substrates and inhibitors that are commonly used in membrane vesicle assays for human $A B C$ transporters, we suggest that $A B C C$-type endogenous proteins in the S. frugiperda genome might be responsible for the observed ATP-dependent transport.

\section{Materials and methods}

\subsection{Materials}

$\mathrm{N}$-methyl quinidine (NMQ) was purchased from SOLVO Biotechnology (Szeged, Hungary) and tritiumlabeled estrone sulfate $\left(\left[{ }^{3} \mathrm{H}\right]-\mathrm{E}_{1} \mathrm{~S}, 54 \mathrm{Ci} / \mathrm{mmol}\right)$ was from PerkinElmer (Waltham, MA, USA). The randomly methylated $\beta$-cyclodextrin-cholesterol complex (RAMEB-cholesterol) was from CycloLab Ltd. (Budapest, Hungary). All water used for preparing solutions was of ultrapure grade. Unless otherwise noted, all other chemicals, including 5(6)-carboxy-2,'7'-dichlorofluorescein (CDCF), estradiol-17 $\beta$-glucuronide $\left(E_{2} 17 G\right)$ and unlabeled $E_{1} S$ used for the transport assays, were from Sigma-Aldrich (St. Louis, MO, USA).

\subsection{Cell culture and vesicle preparation}

Sf9 cells were cultured in suspension at $27{ }^{\circ} \mathrm{C}$ in HyClone SFX insect cell culture medium (GE Healthcare, Little Chalfont, UK) supplemented with $5 \%$ bovine serum albumin (Gibco, Invitrogen, NY, USA). Cells were harvested at a cell density of $2-4 * 10^{6}$ cells $/ \mathrm{ml}$ by centrifugation (1000 $\mathrm{g}$ for $10 \mathrm{~min}$ ) and washed once with 
phosphate buffered saline (PBS). Cell pellets were frozen in liquid nitrogen and stored at $-80{ }^{\circ} \mathrm{C}$ until vesicles were prepared. Membrane vesicles were produced from the harvested cells as described in Sjöstedt et al. (2017b). In short, after two washes with buffer (50 mM Tris, 300 mM mannitol, pH 7), cells were resuspended in membrane buffer (50 mM Tris, $50 \mathrm{mM}$ mannitol, $2 \mathrm{mM} \mathrm{EGTA}, \mathrm{pH}$ 7) and homogenised using a low clearance Dounce homogenizer. Remaining whole cells and larger cell organelles were removed by centrifugation (1200 g, $10 \mathrm{~min}$ ). The supernatant was centrifuged for $75 \mathrm{~min}$ at 100,000 g to collect the crude membrane fraction. This fraction was resuspended in membrane buffer and passed through a 27G needle 20 times to form a homogeneous vesicle suspension. The protein concentration of the vesicle suspension was measured using the Bio-Rad Protein assay (Bio-Rad Laboratories, Hercules, CA, USA). The vesicles ( $\mathrm{Sf} 9+\mathrm{Chol}$ ) were loaded with cholesterol as described in Sjöstedt et al. (2017b) by incubating them on ice in the presence of the water-soluble RAMEB-cholesterol (cholesterol concentration $2.5 \mathrm{mM}$ in the solution) for $20 \mathrm{~min}$. Mock-treated vesicles (Sf9-Mock) were incubated in the absence of cholesterol, but otherwise treated as the cholesterol loaded vesicles. Ready-to-use membrane vesicles were stored at -80 ${ }^{\circ} \mathrm{C}$.

\subsection{Transport assays}

For the transport assays, vesicles suspensions were diluted in buffer containing $40 \mathrm{mM}$ MOPS-Tris (pH 7.0), $60 \mathrm{mM} \mathrm{KCl}$ and $6 \mathrm{mM} \mathrm{MgCl}_{2}$. In each reaction, $50 \mu \mathrm{g}$ of vesicles were incubated at $37^{\circ} \mathrm{C}$ (or $32^{\circ} \mathrm{C}$ for $\mathrm{E}_{1} \mathrm{~S}$ studies (Pal et al., 2007)) with the substrate in the presence and absence of $4 \mathrm{mM}$ ATP. A mixture of labelled and non-labelled $\mathrm{E}_{1} \mathrm{~S}$ was used with a total activity of $42 \mathrm{nCi} /$ well. The final reaction volume in all assays was $75 \mu \mathrm{l}$. In the inhibition assays, an inhibitor was also included at different concentrations. The total DMSO concentration was no more than $2 \%$ in all assays. After incubation with ATP or plain buffer, the reactions were stopped with ice-cold wash buffer ( $40 \mathrm{mM}$ MOPS-TRIS, $70 \mathrm{mM} \mathrm{KCl}, \mathrm{pH}$ 7), filtered on a glassfiber 1.0/0.65 $\mu \mathrm{m}$ MultiScreen-HTS filter plate (Merck Millipore, Burlington, MA, USA) and washed five times. In CDCF assays, vesicles were lysed with $100 \mu \mathrm{l} 0.1 \mathrm{M} \mathrm{NaOH}$ and the amount of CDCF in the elute was measured by fluorometry using Varioskan Flash (Thermo Scientific, Vantaa, Finland) at $510 \mathrm{~nm}$ excitation and $535 \mathrm{~nm}$ emission. Vesicles from NMQ and $E_{2} 17 G$ assays were lysed, and the content eluted with 3:1 $\mathrm{MeOH} / \mathrm{H}_{2} \mathrm{O}$ and analysed as described in Sjöstedt et al. (2017a) and Järvinen et al. (2017), respectively. $\mathrm{E}_{1} \mathrm{~S}$ was analysed by adding $50 \mu$ l Optiphase Hisafe 3 (Perkin Elmer) scintillation liquid to the wells of the filter plate and measuring activity with the Wallac 1450 Microbeta Trilux scintillation counter (Perkin Elmer, Waltham, MA, USA ).

\subsection{Data analysis}


Data are presented as mean with standard deviation (S.D.) from two independent studies performed in triplicate. All individual data points were used in the statistical analysis. The unpaired t-test was used to evaluate significant differences between transport in the presence and absence of ATP. $p$-values $<0.05$ were considered statistically significant. For CDCF kinetics, ATP-dependent transport was calculated as the difference between accumulation in the vesicles in the presence and absence of ATP. Nonlinear regression in GraphPad Prism version 6.05 (GraphPad Software Inc., San Diego, CA, USA) was used to calculate the maximal transport velocity $\left(\mathrm{V}_{\max }\right)$ and the concentration require to reach $50 \% \mathrm{~V}_{\max }\left(\mathrm{K}_{\mathrm{m}}\right)$ transport assuming Michaelis-Menten kinetics. For the inhibition studies, results are presented as relative transport values (\%), where the ATP-dependent transport in the presence of the inhibitor was normalized to the observed transport in assays with vehicle (DMSO) only.

\subsection{Sequence data, sequence, phylogenetic and structural analysis}

The human ABCC2 sequence (UniProt accession no. Q92887) was used as query in NCBI BLAST (http://blast.ncbi.nlm.nih.gov/Blast.cgi; (Altschul et al., 1990)) search against non-redundant sequences in the genome of $S$. frugiperda. The sequences with significant E-value $(<0.001)$ and query coverage of higher than $95 \%$ were collected and aligned using the program MALIGN in the BODIL modeling environment (Lehtonen et al., 2004) using STRMAT110 scoring matrix with a gap penalty of 40 . The percentage identities of the $12 \mathrm{~S}$. frugiperda $\mathrm{ABCC} 2$ transporter sequences were within the range of $98.6-99.6 \%$ with each other and, thus, the previously characterized S. frugiperda $A B C C 2$ sequence ( $S F A B C C 2$ ) with an accession code of ASA45739.1 was selected as a representative SfABCC2 (Banerjee et al., 2017). Furthermore, the $S$. frugiperda $A B C C 3$ sequence with a sequence identity of $54 \%$ to $S f A B C C 2$ was acquired as a result of the BLAST search. To find the ABCC2 and ABCC3 sequences of Spodoptera exigua and Spodoptera litura, the selected SfABCC2 and SfABCC3 sequences were used as a query in the NCBI BLAST searches against nonredundant sequences in these genomes. For both genomes, the top ranked sequences were selected for further studies.

The SfABCC2 sequence was also used as a query in NCBI BLAST ((http://blast.ncbi.nlm.nih.gov/Blast.cgi; (Altschul et al., 1990)) search against Protein Data Bank (PDB) to find crystal structures of homologous proteins. As a result, three-dimensional (3D) structures of bovine MRP1 were found ((Johnson and Chen, 2017, 2018); PDB codes 5uj9, 5aju, 6bhu) and downloaded from PDB. As the 3D structures lack a significant portion of the residues, the bovine $A B C C 1$ sequence was obtained from UniProtKB for sequence alignments. All subsequent sequence alignments were done using MALIGN in the BODIL modeling environment (Lehtonen et al., 2004) and formatted using ESPript 3.0 (Robert and Gouet, 2014). To create a multiple sequence alignment, the sequences for human ABCC1- 6 and ABCC10 (MRP7) were obtained from 
UniProtKB (Jain et al., 2009). The sequence of bovine $A B C C 1$, human ABCC1-3, ABCC6 and ABCC10 with the N-terminal TMDO domain were first aligned and then human ABCC4-5 and ABCC transporters from S. frugiperda, S. litura and S. exiqua were aligned to the previously pre-aligned sequence alignment (Fig. A.1). The sequences used in the study are listed in Table A.1 and the sequence identities based on Fig. A.1 in Table A.2.

To analyze the features of SFABCC2 and SFABCC3 in more detail, the sequences were aligned with the structurally known bovine $A B C C 1$ and the human $A B C C 1-4$ sequences (Fig. 4A). Phylogenetic trees (Fig. 4B and Fig. A.2) were constructed in MEGA7 (Kumar et al., 2016) using the Maximum Likelihood (ML) method based on the Le and Gascuel (2008) model (LG substitution matrix) with gamma distributions (G; Fig. 4B) and with gamma distributions and invariant sites (G+l; Fig. A.2). Complete elimination of gaps and missing data was applied to exclude highly variable regions from analysis and bootstrapping (500 replications) was used to evaluate branch support (Felsenstein, 1985). The domain architecture of the SfABCC2 sequence was analyzed with the Simple Modular Architecture Research Tool (SMART) (Letunic et al., 2012; Schultz et al., 1998) to find domain boundaries. The 3D model for SfABCC2 was created by iTASSER (Roy et al., 2010; Yang et al., 2015; Zhang, 2008) using the pairwise alignment of SfABCC2 (Fig.4; $30.6 \%$ sequence identity) and the template structure of bovine ABCC1 in complex with Mg2+ and ATP (PDB code 1bhu) (Fig. 5). The 3D structures of bovine ABCC1 and the SfABCC2 model were analyzed and visualized with PyMOL (Schrödinger, LCC).

\section{Results}

\subsection{Endogenous ATP-dependent transport in Sf9 vesicles}

The ATP-dependent transport of four commonly used $A B C$ transporter probe substrates $\left(C D C F, E_{2} 17 G, E_{1} S\right.$ and $\mathrm{NMQ}$ ) was tested at concentrations typically used in vesicle transport assays in cholesterol-loaded (Sf9+Chol) and mock-treated Sf9 vesicles (Sf9-Mock) (Fig. 1). Some ATP-dependent transport of CDCF was observed in the mock vesicles without added cholesterol while active transport was not evident for any of the other tested substrates. In contrast, in the cholesterol-loaded vesicles, ATP-dependent transport was observed for all substrates except $E_{1} S$. The clearest effect was seen for CDCF, which had a transport ratio of $7.86 \pm 0.72$ in the $\mathrm{Sf9}+\mathrm{Chol}$ vesicles. The transport ratios of all compounds are shown in Table 1.

Table 1. Transport ratios of commonly used ABC transporter substrates in control vesicles (Sf9-Mock) and cholesterol-loaded Sf9 vesicles (Sf9+Chol).

\begin{tabular}{lll}
\hline Substrate & Transport ratio in & Transport ratio in \\
& Sf9-Mock $( \pm$ S.D.) & Sf9+Chol ( \pm S.D. $)$
\end{tabular}




\begin{tabular}{lll}
\hline $5(6)$-carboxy-2,'7'-dichlorofluorescein (CDCF) & $2.77( \pm 0.68)$ & $7.86( \pm 0.72)$ \\
Estradiol-17ß-glucuronide $\left(\mathrm{E}_{2} 17 \mathrm{G}\right)$ & $1.38( \pm 0.63)$ & $2.46( \pm 0.77)$ \\
Estrone sulfate $\left(\mathrm{E}_{1} \mathrm{~S}\right)$ & $1.06( \pm 0.24)$ & $1.19( \pm 0.22)$ \\
$\mathrm{N}$-methyl quinidine (NMQ) & $1.03( \pm 0.34)$ & $1.74( \pm 0.45)$ \\
\hline
\end{tabular}

The transport ratio was calculated as the transport in the presence of ATP divided by transport in the absence of ATP.

\subsection{Kinetics of endogenous CDCF transport in cholesterol loaded Sf9 vesicles}

Although active transport of $E_{2} 17 G$ and $N M Q$ was observed in cholesterol-loaded vesicles, CDCF had the highest ATP-dependent transport ratio (i.e. transport in the presence of ATP vs in the absence of ATP) in the cholesterol-loaded vesicles. Therefore we continued to investigate the transport kinetics of the endogenous transport of CDCF in the Sf9+Chol vesicles. The ATP-dependent transport of CDCF was time and concentration dependent and followed Michaelis-Menten kinetics with a $K_{m}$ of $8.06 \pm 1.11 \mu \mathrm{M}$ and $V_{\max }$ of $32.8 \pm 1.54 \mathrm{pmol} / \mathrm{min} / \mathrm{mg}$ protein (Fig. 2).

\subsection{Inhibition of endogenous CDCF transport in cholesterol loaded Sf9 vesicles}

The inhibitory activity of five common $A B C$ transporter inhibitors was tested on CDCF transport in the Sf9+Chol vesicles (Fig. 3). CDCF transport was inhibited in a dose-dependent manner by the typical ABCC inhibitors benzbromarone and MK-571, but not by the ABCB1 inhibitor verapamil. On the contrary, $100 \mu \mathrm{M}$ verapamil stimulated CDCF transport. The $A B C C 2 / A B C G 2$ inhibitor sulfasalazine also inhibited CDCF transport effectively, but the potent ABCG2 inhibitor Ko143 showed only a low level of inhibition.

\subsection{Identification and analysis of $S$. frugiperda $A B C C$ transporters}

The sequence similarity search with human $A B C C 2$ protein as a query identified several $A B C C$ transporter sequences in the $S$. frugiperda genome, which could be potential candidates for the observed transport. Apart from $S f A B C C 3$, most of the found the sequences were highly similar (>98 \% sequence identity) to the previously identified SfABCC2 (Banerjee et al., 2017) and can be considered as the same transporter. Thus, SfABCC2 (Banerjee et al., 2017) and SfABCC3 (54\% sequence identity) were selected as representative sequences and used for further analysis. Both $S f A B C C 2$ and $S f A B C C 3$ are expressed in Sf9 cells (Fig. A.3 and $L i$ et al. 2017). The multiple sequence alignment (Fig. A.1, Table A.1) revealed that the ABCC transporters from S. frugiperda, S. litura and S. exiqua share the highest identity to human ABCC4 (39.4-39.8\%; Table A.2) and have a lower identity to the other human ABCC transporters (26.6-31.8\%; Table A.2). In fact, the $S$. litura transporter sequence with the identity of $39.6 \%$ to human $A B C C 4$ is annotated as an ABCC4 protein, while its orthologues in S. frugiperda and S. exigua are annotated as ABCC2 despite also having the highest 
sequence identity with human ABCC4. Based on the SMART analysis (Letunic et al., 2012; Schultz et al., 1998), the $S$. frugiperda ABCC transporters were predicted to consist of two TMDs (SfABCC2: residues 107383 and 758-1046) and two intracellular NBDs (SfABCC2: residues 495-668 and 1121-1301). In the 3D model of SfABCC2, which was modeled in the outward-facing conformation, the TMD1 consists of residues 96-404 and TMD1 of residues 755-1072 and the NBD1 includes residues 405-712 and NDB2 has residues 1073-1314 (Fig. 5A). The SfABCC2 model had a C-score of 0.06 that gives a good confidence for the quality of the 3D model created by I-TASSER (ranges between -5 - 2 with higher value being better) and a TM-score of $0.72 \pm 0.11$, which shows the topological similarity between the template and the model (TM-score $>0.5$ approximate cutoff value) (Roy et al., 2010). Analysis of the bovine ABCC1 crystal structure in complex with ATP and $\mathrm{Mg}^{2+}$ reveals that the ATP- and $\mathrm{Mg}^{2+}$-binding residues are highly conserved in the studied proteins and, thus the two S. frugiperda ABCC transporters have two binding sites for ATP and $\mathrm{Mg}^{2+}$ (Fig. 4; marked with green and magenta asterisks, respectively). The phylogenetic analysis shows that the two $S$. frugiperda $A B C C$ transporters are most closely related to the human ABCC4 protein (Fig. $4 B$ and Fig. A.2). In the 3D model for SfABCC2, the residues from Walker A and B motif from one NDB forms the binding site together with the signature motif of the other NDB (Fig. 5B-C). Thr510, QIn538 and Asp617, coordinate one of the Mg2+ ions (Fig. 5B), whereas the other Mg2+ is bound by Ser1136, Qln1176 and Asp1251 (Fig. 5C). All these residues, expect Thr510, (a serine in bovine ABCC1) are totally conserved. Similarly, the ATP binding sites are extremely conserved in SfABCC2 (Figs. 4 and $5 B-C$ ). Altogether, the results indicate that SfABCC2 is a functional $A B C$ transporter.

\section{Discussion}

We found endogenous ATP-dependent transport in vesicles from S.frugiperda, which was activated when cholesterol was added to the vesicles. Cholesterol-loading is used to improve the activity of cholesterolsensitive human ABC transporters such as ABCG2 and ABCB1 (Heredi-Szabo et al., 2013; Pal et al., 2007; Telbisz et al., 2007), while the ABCC transporters are most often studied in untreated vesicles. Endogenous transport in Sf9+Chol vesicles was noted for several probe substrates that are commonly used to study human $A B C$ transporters in drug development. $A B C G 2$ substrate $E_{1} S$ (Imai et al., 2003) did not show significant ATP-dependent uptake in Sf9 vesicles, and only low cholesterol-induced background transport was observed here for $\mathrm{ABCB} 1$ substrate $\mathrm{NMQ}$, in line with a previous report (Heredi-Szabo et al., 2013). Transport of $E_{2} 17 G$ and CDCF was, however, more clearly affected by cholesterol-loading. Although they are typically used as ABCC2 probe substrates (Brouwer et al., 2013; Heredi-Szabo et al., 2008), $E_{2} 17 G$ and CDCF are also transported by multiple other human $A B C$ transporters, including several other $A B C C$ family members, namely ABCC3, 4 and 5 (Borst et al., 2007; Järvinen et al., 2017; Pratt et al., 2006; Seelheim et al., 
2013). In Sf9+Chol vesicles, ATP-dependent CDCF transport was 6.6-fold higher than in vesicles without cholesterol loading. The transport could be inhibited by typical $A B C C$ inhibitors, but was not affected by inhibitors of $A B C G 2$ and $A B C B 1$. The highest concentration of verapamil tested stimulated $C D C F$ transport in the $\mathrm{Sfg}+\mathrm{CHOL}$ vesicles. Interestingly, verapamil has previously been reported to stimulate CDCF transport in Sf9-ABCC2 vesicles as wells as coproporphyrin I transport in Sf9 vesicles overexpressing rat Abcc2 (Gilibili et al., 2018; Munic et al., 2011). Based on the substrate and inhibitor preferences of the endogenous, cholesterol-activated transport, we speculate that it is caused by an ABCC-type transporter (or transporters) of S. frugiperda. Though it is not typically used, cholesterol-loading is known to activate ABCC2-dependent transport of some substrates (Guyot et al., 2014; Ito et al., 2008; Paulusma et al., 2009) including a moderate increase in the ATP-dependent transport of CDCF (unpublished data).

The expression and function of insect $A B C$ transporters has mainly been studied in Drosophila melanogaster. As an example of this, it is known that the product of the Drosophila gene white, plays a role in the pigmentation of fruit fly eyes (Mackenzie et al., 1999). Its human homologue ABCG1 is involved in cholesterol and phospholipid transport in macrophages (Klucken et al., 2000) and based on sequence identity $A B C G 1$ is the closest relative to human $A B C G 2$. However, D. melanogaster also express an orthologue of the human MRPs that can transport typical $A B C C$ substrates $E_{2} 17 G, C D C F$ and leukotriene $C 4$ (Szeri et al., 2009). Although the genome of $S$. frugiperda is not as thoroughly studied as the $D$. melanogaster, there is evidence of $A B C$ transporter expression in S. frugiperda: A truncated mutant form of $S f A B C C 2$ has been identified as a cause for resistance of $S$. frugiperda to the insecticidal protein produced by genetically modified Bt corn (Banerjee et al., 2017). In S. frugiperda, ABCC2 acts as a receptor for the toxic protein, but the interaction is disturbed in the mutant form.

In this study, using the human $A B C C 2$ gene sequence, a BLAST search was performed to identify $A B C C$ type genes in the $S$. frugiperda genome as potential candidates for the endogenous transport activity observed in the Sf9 vesicles. This lead to the identification of two previously annotated genes of the ABCC-type ( $S f A B C C 2$ and $S f A B C C 3$ ), but no new candidate genes. The expression of these genes in Sf9 cells was verified using reverse transcription polymerase chain reaction (RT-PCR) (Fig. A.3). Based on sequence analysis, these genes encode for a protein with two TMDs and two NBDs and appear to be functional transporters, as the residues involved in $\mathrm{Mg}^{2+}$ and ATP binding are conserved. Interestingly, unlike human $\mathrm{ABCC}$, these $S$. frugiperda genes do not code for a third $T M D$, typically named $\mathrm{TMD}_{0}$, which is characteristic to human $A B C C 2$, as well as $A B C C 1$ and 3, but is lacking from human $A B C C 4$. In fact, the sequence identity of the $S f A B C C 2$ gene is higher to human ABCC4 (40.7\%) than to human ABCC2 (32.4\%). However, human ABCC4 showed no active uptake of CDCF in vesicles derived from ABCC4 overexpressing HEK cells (Pratt et al., 2006). Interestingly, both the $S f A B C C 2$ and $S f A B C C 3$ genes are found in two close relatives to $S$. frugiperda, 
S. litura and S. exiqua, but in S.litura, the $A B C C 2$ gene has been annotated as $A B C C 4$. In humans $A B C C 2$ and $A B C C 4$ share substrates, but have distinct localization in the liver (Hillgren et al., 2013). Unlike ABCC2 that is involved in biliary excretion, $A B C C 4$ is expressed on the basolateral membranes of hepatocytes. ABCC4 is upregulated in cholestatic conditions and serves as a type of safety valve to help excrete accumulating bile acids (Gradhand et al., 2008; Keitel et al., 2005).

An interesting finding in this study was that the observed activity of endogenous $S$. frugiperda transport was stimulated by the addition of cholesterol to the membrane vesicles. Enhancement of transport activity after cholesterol loading has previously been observed for human ABC transporters (Eckford and Sharom, 2008; Fenyvesi et al., 2008; Guyot et al., 2014; Pal et al., 2007; Paulusma et al., 2009; Telbisz et al., 2007), which also supports our hypothesis that the endogenous transport in S. frugiperda vesicles is mediated by an $A B C$ transporter. The exact mechanism of how cholesterol stimulates $A B C$ transporter activity is still unknown. It has been suggested that a rigid membrane environment might be important for supporting the correct transporter conformation (Sharom, 2014) or that cholesterol might bind directly to the transporter since it is structurally similar to other steroidal $A B C$ transporter substrates (Guyot et al., 2014). In fact, the cryo-electron microscopy based structure of human ABCG2 shows cholesterol bound to the binding pocket of the transporter (Taylor et al., 2017). Additionally, for ABCB1, it has been suggested that a cholesterol-rich environment can enhance the partitioning of $A B C B 1$ substrates into the lipid bilayer and allow them easier access to the substrate binding site (Eckford and Sharom, 2008). However, for the hydrophilic substrates studied here, a direct interaction of the transporter with cholesterol or increased transporter stability seem to be the more plausible explanations.

In addition to the positive effects of cholesterol-loading on the ATP-dependent transport of the three compounds reported here, in a milder fashion, cholesterol also activates the transport of selected glucuronidated compounds in control Sf9 vesicles (e.g. 4-methylumbelliferone and estrogens) (Järvinen et al., 2018; Järvinen et al., 2017). Furthermore, ochratoxin was reported by Guyot et al. (2014) to be transported by endogenous transporters in Sf21 membrane vesicles. Similar to our findings, the authors suggest that endogenous transport is mediated by an ABCC-type transporter, because the transport was inhibited in part by MK-571 and not by the ABCB1 inhibitor PSC833. The endogenous ochratoxin transport was, however, not activated by cholesterol. Cholesterol effects on endogenous and exogenous transporters could be substrate-dependent as shown by Guyot et al. (2014). In Sf21-ABCC2 vesicles $V_{\max }$ was increased and $K_{m}$ decreased by cholesterol loading for both $E_{2} 17 G$ and cholecystokinin octapeptide (CCK8) transport, but in addition, the transport of $E_{2} 17 G$ changed from co-operative binding to Michaelis-Menten kinetics. Interestingly, based on our results these changes in $E_{2} 17 \mathrm{G}$ transport might be partly due to increased endogenous transport which was not characterized by Guyot et al. (2014), but was shown to be influenced 
by cholesterol in the present study.

Taking together our in vitro observations and the presence of $A B C C$ type genes in the $S$. frugiperda genome, it is advisable that in addition to ATP-deficient conditions, Sf9 vesicles that do not overexpress human transporters are included as controls when using the Sf9 expression system for studying $A B C$ drug transporters, as was previously suggested by Brouwer et al. (2013). This is especially important when studying new potential substrates using cholesterol-loaded vesicles, although it should be noted that some endogenous transport was observed for CDCF even without additional cholesterol. Even low background transport levels may make it difficult to verify transport of low activity substrates. For CDCF, however, the endogenous transport is much lower (30- to 40-fold) than that observed in ABCC2 overexpressing Sf9 vesicles (Kidron et al., 2012). At conditions used for inhibition studies in our laboratory and elsewhere, transport ratios in the presence of human $A B C$ transporters for the substrates used in this study are typically more than ten-fold (Elsby et al., 2011; Heredi-Szabo et al., 2013; Pedersen et al., 2008).

In conclusion, we show here that cholesterol-loading of Sf9-derived membrane vesicles stimulates endogenous ATP-dependent transport in these vesicles. The results of this study highlight that although insect-based expression systems can provide useful transporter-specific information, stringent controls must be included to avoid false positives, especially when low transport activity is observed. $A B C$ transporters expressed by $S$. frugiperda are not as well characterized as human $A B C$ transporters, but their potential presence in Sf9-derived vesicles should not be overlooked. After characterizing cholesterolactivated endogenous transport in Sf9 vesicles with typical human $A B C$ transporter substrates and inhibitors, we bring forward the hypothesis that the observed transport is mediated by an ABCC-type transporter. Based on sequence analysis, we identified two candidate genes (SfACC2 and SfABCC3) in the $S$. frugiperda genome, but further studies are needed to confirm whether they are responsible for the observed endogenous transport.

\section{Acknowledgements}

We thank Leena Pietilä and Estelle Pierrot for technical assistance and Erkka Järvinen for help with the HPLC analysis. The Drug Discovery and Chemical Biology Network, funded by Biocenter Finland, is acknowledged for providing access to the screening instrumentation (Varioskan). Instruct-Fl and Biocenter Finland are acknowledged for the infrastructure support in bioinformatics (J.V. Lehtonen), translational activities and structural biology and CSC IT Center for Science for laboratory and computational infrastructure support at the Structural Bioinformatics Laboratory, Åbo Akademi University. Prof. Mauno 
Vihinen is acknowledged for the support and comfortable working environment during TAS research visits at the Department of Experimental Medical Science, Lund University, Sweden. This study was funded by the University of Helsinki Doctoral Programme in Drug Research (NS), the Academy of Finland (NS, HK), the Sigrid Juselius Foundation (TAS), Åbo Akademis Jubileumfond 1968 (TAS) and Tor, Joe, and Pentti Borg's Foundation (TAS).

\section{Legends to figures}

Fig. 1. Endogenous transport of $A B C$ transporter substrates typically used in vesicular transport assays. The transport of 5(6)-carboxy-2,'7'-dichlorofluorescein CDCF (5 $\mu \mathrm{M}, 30 \mathrm{~min})$, estradiol-17 $\beta$-glucuronide $\mathrm{E}_{2} 17 \mathrm{G}(10 \mu \mathrm{M}, 5 \mathrm{~min})$, estrone sulfate $\mathrm{E}_{1} \mathrm{~S}(1 \mu \mathrm{M}, 2 \mathrm{~min})$ and $\mathrm{N}$-methyl quinidine $\mathrm{NMQ}(2 \mu \mathrm{M}, 3 \mathrm{~min})$ was studied in Sf9+Chol and Sf9-Mock vesicles in the presence (black bars) and absence (white bars) of ATP. Data is presented as mean \pm S.D. ( $n=2$ in triplicate). Statistical significance of differences between + ATP and -ATP conditions was analyzed using the unpaired t-test. ${ }^{*} p<0.05,{ }^{* * *} p<0.001$.

Fig. 2. Transport of 5(6)-carboxy-2,'7'-dichlorofluorescein (CDCF) in Sf9 membrane vesicles. A. Time dependence of CDCF transport in Sf9+Chol vesicles in the presence (closed circles) and absence (open circles) of ATP. Concentration of CDCF in the assay was $5 \mu \mathrm{M}$ (representative figure, $\mathrm{n}=1$ in triplicate). B. Concentration dependence of CDCF transport in Sf9+Chol (circles) and Sf9-Mock (squares) vesicles. Transport was studied in the presence (solid symbols) or absence (open symbols) of ATP for 10 min at 37 ${ }^{\circ} \mathrm{C}$. ATP-dependent uptake of CDCF in Sf9+Chol vesicles (grey solid circles) was calculated by subtracting these two transport values. The grey line shows curve fitting to the Michaelis-Menten equation $\left(K_{m}=8.06 \pm\right.$ $1.11 \mu \mathrm{M}$ and $V_{\max }=32.8 \pm 1.54 \mathrm{pmol} / \mathrm{min} / \mathrm{mg}$ protein) in GraphPad Prism. Data is shown as mean \pm S.D. (n $=2$ in triplicate [Sf9+Chol] or $n=1-2$ in triplicate [Sf9-Mock]).

Fig. 3. Inhibition of 5(6)-carboxy-2,'7'-dichlorofluorescein (CDCF) transport in Sf9+Chol vesicles. Inhibition experiments were performed using inhibitors of $A B C C s$ (benzbromarone, MK-571), ABCC/ABCG2 (sulfasalazine), ABCG2 (Ko143) and ABCB1 (verapamil). Sf9+Chol vesicles were incubated with $5 \mu \mathrm{M}$ CDCF for $30 \mathrm{~min}$ at $37^{\circ} \mathrm{C}$ with the inhibitor at 1,10 or $100 \mu \mathrm{M}$. Transport activity is presented as relative ATPdependent transport activity (\%), which is the observed transport in the presence of inhibitor normalized to the transport activity in the absence of inhibitor. Bars show the mean \pm S.D. ( $n=2$ in triplicate). 
Fig. 4. Sequence alignment and phylogenetic analysis of $A B C C$ transporters. A. Sequence alignment. The secondary structure elements in the bovine $A B C C 1$ structure are shown above the alignment. The positions of the Walker A/B and Signature motif are labeled and the residues involved in ATP and $\mathrm{Mg}^{2+}$ binding are marked with green and magenta asterisks, respectively. All these positions are highly conserved in the sequences of $S$. frugiperda $A B C C$ transporters. B. Consensus $M L$ tree. The phylogenetic tree confidently shows (bootstrap support of $100 \%$ ) that $S f A B C C 2$ and $S f A B C C 3$ are evolutionarily most closely related to human ABCC4.

Fig. 5. 3D model for SfABCC2. A. The overall fold of $S f A B C C 2$. The ATP binding site in NBD1 B. and NBD2 C. are highly conserved in SfABCC2. The residues from the lasso area are colored in orange, NDB1 in pink and NDB2 in violet. TMD1 is shown in pink and TMD2 in violet. The NDB1 domain is colored beige and NDB2 blue. The residues involved in binding the ATP molecules (yellow space-filing model) are colored green and those that coordinate the $\mathrm{Mg}^{2+}$ ions (cyan spheres) in magenta.

\section{References}

Ahlin, G., Hilgendorf, C., Karlsson, J., Szigyarto, C.A., Uhlen, M., Artursson, P., 2009. Endogenous gene and protein expression of drug-transporting proteins in cell lines routinely used in drug discovery programs. Drug Metab Dispos 37, 2275-2283.

Altschul, S.F., Gish, W., Miller, W., Myers, E.W., Lipman, D.J., 1990. Basic local alignment search tool. J Mol Biol 215, 403-410.

Banerjee, R., Hasler, J., Meagher, R., Nagoshi, R., Hietala, L., Huang, F., Narva, K., Jurat-Fuentes, J.L., 2017. Mechanism and DNA-based detection of field-evolved resistance to transgenic Bt corn in fall armyworm (Spodoptera frugiperda). Sci Rep 7, 10877.

Borst, P., de Wolf, C., van de Wetering, K., 2007. Multidrug resistance-associated proteins 3, 4, and 5. Pflugers Arch 453, 661-673.

Brouwer, K.L., Keppler, D., Hoffmaster, K.A., Bow, D.A., Cheng, Y., Lai, Y., Palm, J.E., Stieger, B., Evers, R., International Transporter, C., 2013. In vitro methods to support transporter evaluation in drug discovery and development. Clin Pharmacol Ther 94, 95-112.

Dean, M., Rzhetsky, A., Allikmets, R., 2001. The human ATP-binding cassette (ABC) transporter superfamily. Genome Res 11, 1156-1166.

Eckford, P.D., Sharom, F.J., 2008. Interaction of the P-glycoprotein multidrug efflux pump with cholesterol: effects on ATPase activity, drug binding and transport. Biochemistry 47, 13686-13698.

Elsby, R., Smith, V., Fox, L., Stresser, D., Butters, C., Sharma, P., Surry, D.D., 2011. Validation of membrane vesicle-based breast cancer resistance protein and multidrug resistance protein 2 assays to assess drug transport and the potential for drug-drug interaction to support regulatory submissions. Xenobiotica 41 , 764-783.

Felsenstein, J., 1985. Confidence Limits on Phylogenies: An Approach Using the Bootstrap. Evolution 39, 783-791. 
Fenyvesi, F., Fenyvesi, E., Szente, L., Goda, K., Bacso, Z., Bacskay, I., Varadi, J., Kiss, T., Molnar, E., Janaky, T., Szabo, G., Jr., Vecsernyes, M., 2008. P-glycoprotein inhibition by membrane cholesterol modulation. Eur J Pharm Sci 34, 236-242.

Giacomini, K.M., Huang, S.M., Tweedie, D.J., Benet, L.Z., Brouwer, K.L., Chu, X., Dahlin, A., Evers, R., Fischer, V., Hillgren, K.M., Hoffmaster, K.A., Ishikawa, T., Keppler, D., Kim, R.B., Lee, C.A., Niemi, M., Polli, J.W., Sugiyama, Y., Swaan, P.W., Ware, J.A., Wright, S.H., Yee, S.W., Zamek-Gliszczynski, M.J., Zhang, L., 2010. Membrane transporters in drug development. Nat Rev Drug Discov 9, 215-236.

Gilibili, R.R., Kurawattimath, V., Murali, B.V., Lai, Y., Mariappan, T.T., Shen, H., Chatterjee, S., 2018. In Vitro Stimulation of Multidrug Resistance-Associated Protein 2 Function Is Not Reproduced In Vivo in Rats. Pharmaceutics 10.

Gimpl, G., Klein, U., Reilander, H., Fahrenholz, F., 1995. Expression of the human oxytocin receptor in baculovirus-infected insect cells: high-affinity binding is induced by a cholesterol-cyclodextrin complex. Biochemistry 34, 13794-13801.

Glavinas, H., Krajcsi, P., Cserepes, J., Sarkadi, B., 2004. The role of ABC transporters in drug resistance, metabolism and toxicity. Curr Drug Deliv 1, 27-42.

Glavinas, H., Mehn, D., Jani, M., Oosterhuis, B., Heredi-Szabo, K., Krajcsi, P., 2008. Utilization of membrane vesicle preparations to study drug-ABC transporter interactions. Expert Opin Drug Metab Toxicol 4, 721732.

Goh, L.B., Spears, K.J., Yao, D., Ayrton, A., Morgan, P., Roland Wolf, C., Friedberg, T., 2002. Endogenous drug transporters in in vitro and in vivo models for the prediction of drug disposition in man. Biochem Pharmacol 64, 1569-1578.

Gottesman, M.M., Fojo, T., Bates, S.E., 2002. Multidrug resistance in cancer: role of ATP-dependent transporters. Nat Rev Cancer 2, 48-58.

Gradhand, U., Lang, T., Schaeffeler, E., Glaeser, H., Tegude, H., Klein, K., Fritz, P., Jedlitschky, G., Kroemer, H.K., Bachmakov, I., Anwald, B., Kerb, R., Zanger, U.M., Eichelbaum, M., Schwab, M., Fromm, M.F., 2008. Variability in human hepatic MRP4 expression: influence of cholestasis and genotype. Pharmacogenomics $J$ $8,42-52$.

Guyot, C., Hofstetter, L., Stieger, B., 2014. Differential effects of membrane cholesterol content on the transport activity of multidrug resistance-associated protein 2 ( $A B C C 2$ ) and of the bile salt export pump (ABCB11). Mol Pharmacol 85, 909-920.

Guyot, C., Stieger, B., 2011. Interaction of bile salts with rat canalicular membrane vesicles: evidence for bile salt resistant microdomains. J Hepatol 55, 1368-1376.

Heredi-Szabo, K., Kis, E., Molnar, E., Gyorfi, A., Krajcsi, P., 2008. Characterization of 5(6)-carboxy-2,'7'dichlorofluorescein transport by MRP2 and utilization of this substrate as a fluorescent surrogate for LTC4. J Biomol Screen 13, 295-301.

Heredi-Szabo, K., Palm, J.E., Andersson, T.B., Pal, A., Mehn, D., Fekete, Z., Beery, E., Jakab, K.T., Jani, M., Krajcsi, P., 2013. A P-gp vesicular transport inhibition assay - optimization and validation for drug-drug interaction testing. Eur J Pharm Sci 49, 773-781.

Higgins, C.F., 1992. ABC transporters: from microorganisms to man. Annu Rev Cell Biol 8, 67-113.

Hillgren, K.M., Keppler, D., Zur, A.A., Giacomini, K.M., Stieger, B., Cass, C.E., Zhang, L., International Transporter, C., 2013. Emerging transporters of clinical importance: an update from the International Transporter Consortium. Clin Pharmacol Ther 94, 52-63.

Imai, Y., Asada, S., Tsukahara, S., Ishikawa, E., Tsuruo, T., Sugimoto, Y., 2003. Breast cancer resistance protein exports sulfated estrogens but not free estrogens. Mol Pharmacol 64, 610-618.

Ito, K., Hoekstra, D., van Ijzendoorn, S.C., 2008. Cholesterol but not association with detergent resistant membranes is necessary for the transport function of MRP2/ABCC2. FEBS Lett 582, 4153-4157. 
Jain, E., Bairoch, A., Duvaud, S., Phan, I., Redaschi, N., Suzek, B.E., Martin, M.J., McGarvey, P., Gasteiger, E., 2009. Infrastructure for the life sciences: design and implementation of the UniProt website. BMC Bioinformatics 10, 136.

Järvinen, E., Deng, F., Kidron, H., Finel, M., 2018. Efflux transport of estrogen glucuronides by human MRP2, MRP3, MRP4 and BCRP. J Steroid Biochem Mol Biol 178, 99-107.

Järvinen, E., Troberg, J., Kidron, H., Finel, M., 2017. Selectivity in the Efflux of Glucuronides by Human Transporters: MRP4 Is Highly Active toward 4-Methylumbelliferone and 1-Naphthol Glucuronides, while MRP3 Exhibits Stereoselective Propranolol Glucuronide Transport. Mol Pharm 14, 3299-3311.

Johnson, Z.L., Chen, J., 2017. Structural Basis of Substrate Recognition by the Multidrug Resistance Protein MRP1. Cell 168, 1075-1085 e1079.

Johnson, Z.L., Chen, J., 2018. ATP Binding Enables Substrate Release from Multidrug Resistance Protein 1. Cell 172, 81-89 e10.

Keitel, V., Burdelski, M., Warskulat, U., Kuhlkamp, T., Keppler, D., Haussinger, D., Kubitz, R., 2005. Expression and localization of hepatobiliary transport proteins in progressive familial intrahepatic cholestasis. Hepatology 41, 1160-1172.

Kidron, H., Wissel, G., Manevski, N., Hakli, M., Ketola, R.A., Finel, M., Yliperttula, M., Xhaard, H., Urtti, A., 2012. Impact of probe compound in MRP2 vesicular transport assays. Eur J Pharm Sci 46, 100-105.

Klucken, J., Buchler, C., Orso, E., Kaminski, W.E., Porsch-Ozcurumez, M., Liebisch, G., Kapinsky, M., Diederich, W., Drobnik, W., Dean, M., Allikmets, R., Schmitz, G., 2000. ABCG1 (ABC8), the human homolog of the Drosophila white gene, is a regulator of macrophage cholesterol and phospholipid transport. Proc Natl Acad Sci U S A 97, 817-822.

Kumar, S., Stecher, G., Tamura, K., 2016. MEGA7: Molecular Evolutionary Genetics Analysis Version 7.0 for Bigger Datasets. Mol Biol Evol 33, 1870-1874.

Le, S.Q., Gascuel, O., 2008. An improved general amino acid replacement matrix. Mol Biol Evol 25, 13071320.

Lehtonen, J.V., Still, D.J., Rantanen, V.V., Ekholm, J., Bjorklund, D., Iftikhar, Z., Huhtala, M., Repo, S., Jussila, A., Jaakkola, J., Pentikainen, O., Nyronen, T., Salminen, T., Gyllenberg, M., Johnson, M.S., 2004. BODIL: a molecular modeling environment for structure-function analysis and drug design. J Comput Aided Mol Des 18, 401-419.

Letunic, I., Doerks, T., Bork, P., 2012. SMART 7: recent updates to the protein domain annotation resource. Nucleic Acids Res 40, D302-305.

Li, J., Ma, Y., Yuan, W., Xiao, Y., Liu, C., Wang, J., Peng, J., Peng, R., Soberon, M., Bravo, A., Yang, Y., Liu, K., 2017. FOXA transcriptional factor modulates insect susceptibility to Bacillus thuringiensis Cry1Ac toxin by regulating the expression of toxin-receptor $A B C C 2$ and ABCC3 genes. Insect Biochem Mol Biol 88, 1-11.

Mackenzie, S.M., Brooker, M.R., Gill, T.R., Cox, G.B., Howells, A.J., Ewart, G.D., 1999. Mutations in the white gene of Drosophila melanogaster affecting $A B C$ transporters that determine eye colouration. Biochim Biophys Acta 1419, 173-185.

Marheineke, K., Grunewald, S., Christie, W., Reilander, H., 1998. Lipid composition of Spodoptera frugiperda (Sf9) and Trichoplusia ni (Tn) insect cells used for baculovirus infection. FEBS Lett 441, 49-52.

Meyer dos Santos, S., Weber, C.C., Franke, C., Muller, W.E., Eckert, G.P., 2007. Cholesterol: Coupling between membrane microenvironment and ABC transporter activity. Biochem Biophys Res Commun 354, 216-221.

Munic, V., Hlevnjak, M., Erakovic Haber, V., 2011. Characterization of rhodamine-123, calcein and 5(6)carboxy-2',7'-dichlorofluorescein (CDCF) export via MRP2 (ABCC2) in MES-SA and A549 cells. Eur J Pharm Sci 43, 359-369. 
Pal, A., Mehn, D., Molnar, E., Gedey, S., Meszaros, P., Nagy, T., Glavinas, H., Janaky, T., von Richter, O., Bathori, G., Szente, L., Krajcsi, P., 2007. Cholesterol potentiates ABCG2 activity in a heterologous expression system: improved in vitro model to study function of human ABCG2. J Pharmacol Exp Ther 321, 1085-1094.

Paulusma, C.C., de Waart, D.R., Kunne, C., Mok, K.S., Elferink, R.P., 2009. Activity of the bile salt export pump (ABCB11) is critically dependent on canalicular membrane cholesterol content. J Biol Chem 284, 9947-9954.

Pedersen, J.M., Matsson, P., Bergstrom, C.A., Norinder, U., Hoogstraate, J., Artursson, P., 2008. Prediction and identification of drug interactions with the human ATP-binding cassette transporter multidrugresistance associated protein 2 (MRP2; ABCC2). J Med Chem 51, 3275-3287.

Pratt, S., Chen, V., Perry, W.I., 3rd, Starling, J.J., Dantzig, A.H., 2006. Kinetic validation of the use of carboxydichlorofluorescein as a drug surrogate for MRP5-mediated transport. Eur J Pharm Sci 27, 524-532.

Robert, X., Gouet, P., 2014. Deciphering key features in protein structures with the new ENDscript server. Nucleic Acids Res 42, W320-324.

Roy, A., Kucukural, A., Zhang, Y., 2010. I-TASSER: a unified platform for automated protein structure and function prediction. Nat Protoc 5, 725-738.

Schultz, J., Milpetz, F., Bork, P., Ponting, C.P., 1998. SMART, a simple modular architecture research tool: identification of signaling domains. Proc Natl Acad Sci U S A 95, 5857-5864.

Seelheim, P., Wullner, A., Galla, H.J., 2013. Substrate translocation and stimulated ATP hydrolysis of human $A B C$ transporter MRP3 show positive cooperativity and are half-coupled. Biophys Chem 171, 31-37.

Sharom, F.J., 2014. Complex Interplay between the P-Glycoprotein Multidrug Efflux Pump and the Membrane: Its Role in Modulating Protein Function. Front Oncol 4, 41.

Sjöstedt, N., Deng, F., Rauvala, O., Tepponen, T., Kidron, H., 2017a. Interaction of Food Additives with Intestinal Efflux Transporters. Mol Pharm 14, 3824-3833.

Sjöstedt, N., Holvikari, K., Tammela, P., Kidron, H., 2017b. Inhibition of Breast Cancer Resistance Protein and Multidrug Resistance Associated Protein 2 by Natural Compounds and Their Derivatives. Mol Pharm 14, 135-146.

Storch, C.H., Ehehalt, R., Haefeli, W.E., Weiss, J., 2007. Localization of the human breast cancer resistance protein (BCRP/ABCG2) in lipid rafts/caveolae and modulation of its activity by cholesterol in vitro. J Pharmacol Exp Ther 323, 257-264.

Szeri, F., Ilias, A., Pomozi, V., Robinow, S., Bakos, E., Varadi, A., 2009. The high turnover Drosophila multidrug resistance-associated protein shares the biochemical features of its human orthologues. Biochim Biophys Acta 1788, 402-409.

Taylor, N.M.I., Manolaridis, I., Jackson, S.M., Kowal, J., Stahlberg, H., Locher, K.P., 2017. Structure of the human multidrug transporter ABCG2. Nature 546, 504-509.

Telbisz, A., Muller, M., Ozvegy-Laczka, C., Homolya, L., Szente, L., Varadi, A., Sarkadi, B., 2007. Membrane cholesterol selectively modulates the activity of the human ABCG2 multidrug transporter. Biochim Biophys Acta 1768, 2698-2713.

Yang, J., Yan, R., Roy, A., Xu, D., Poisson, J., Zhang, Y., 2015. The I-TASSER Suite: protein structure and function prediction. Nat Methods 12, 7-8.

Zhang, Y., 2008. I-TASSER server for protein 3D structure prediction. BMC Bioinformatics 9, 40. 
A.

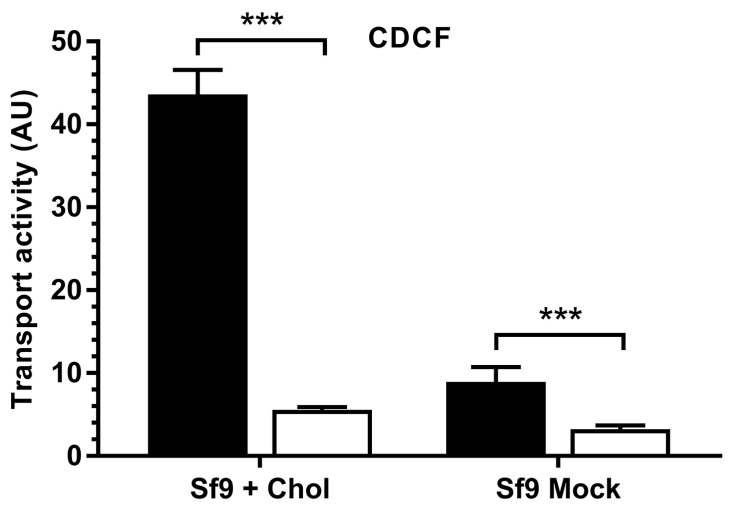

c.

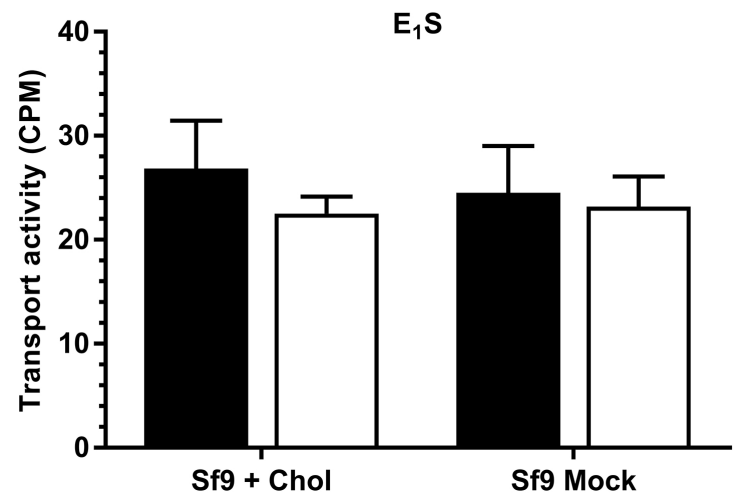

B.

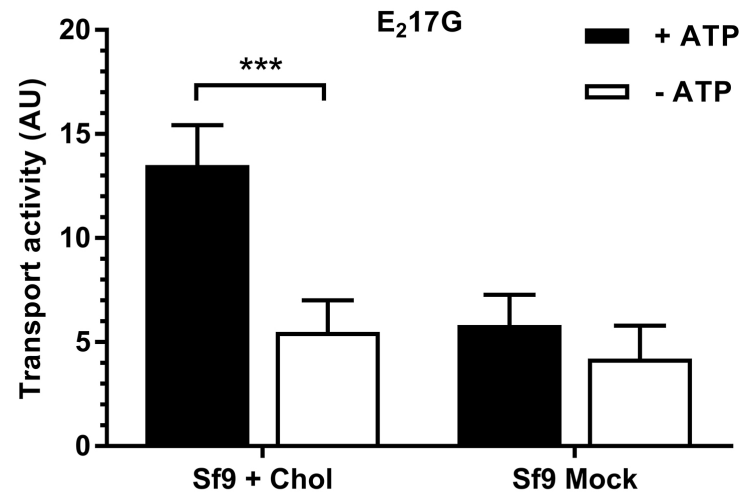

D.

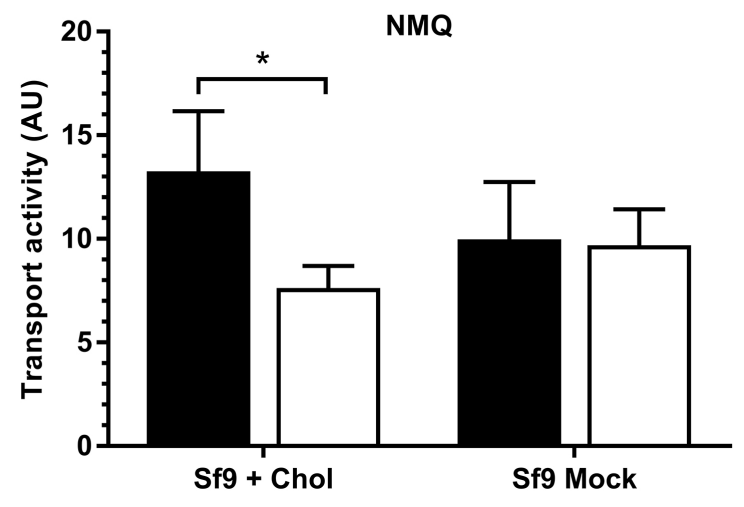


A.
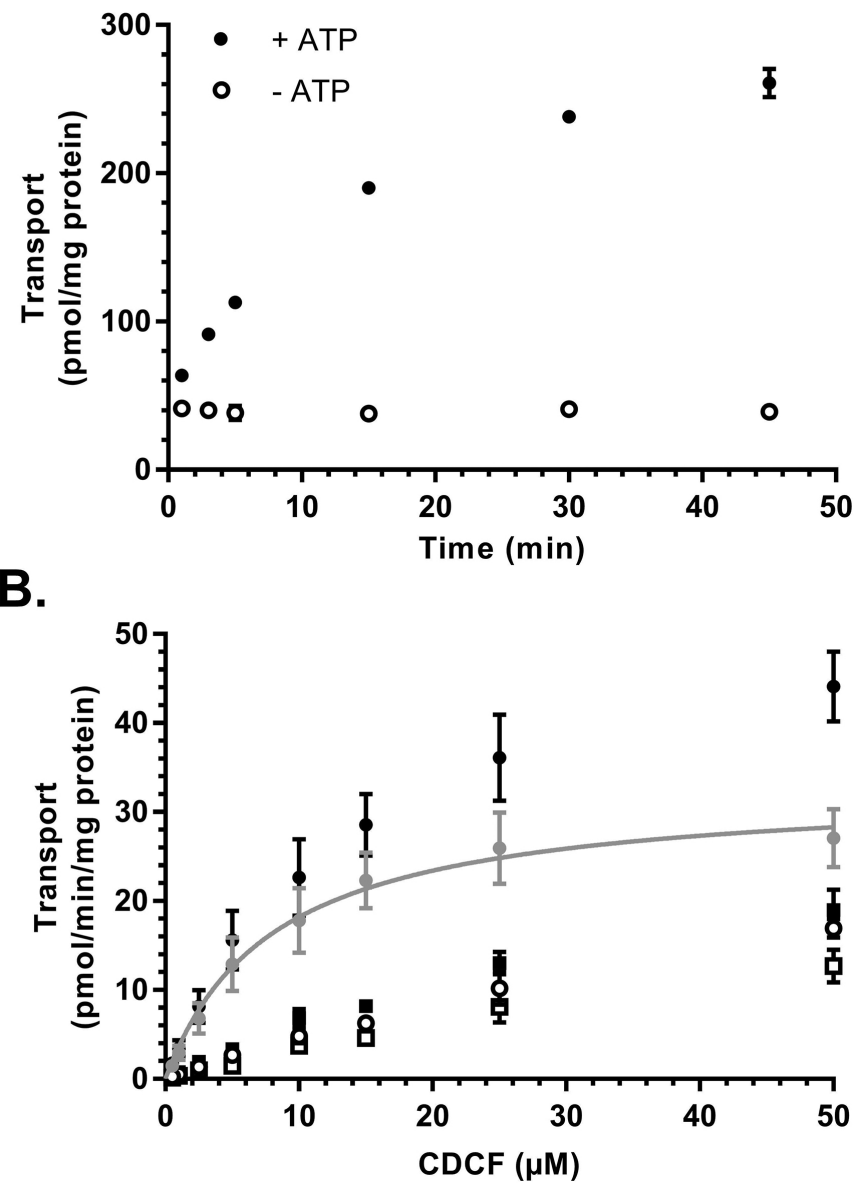

Figure 2 


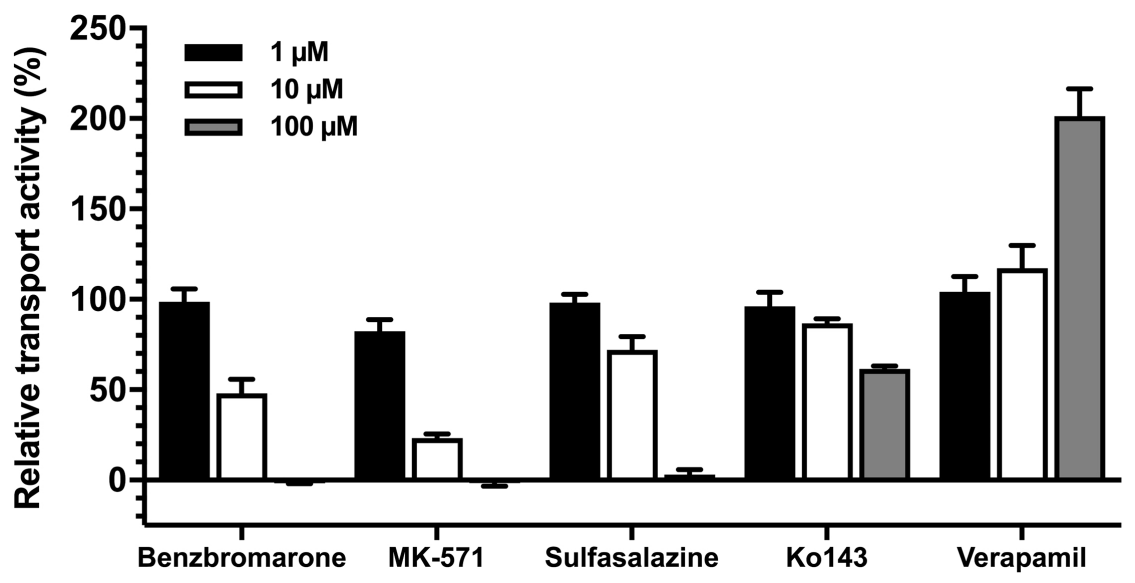

Figure 3 
A $\begin{array}{cccc}\alpha y & \alpha y & \eta 2 & \alpha 10\end{array}$ T T elecereele

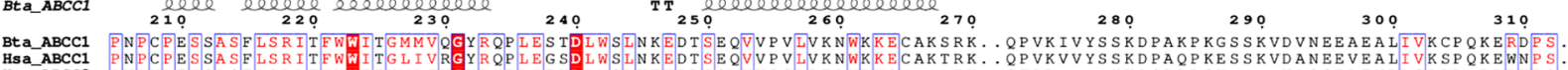

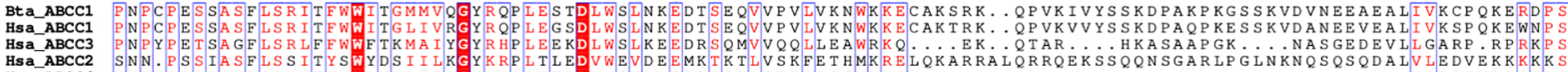

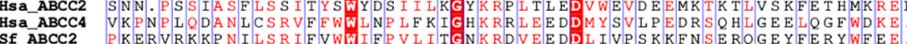

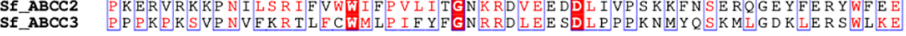

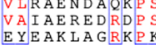

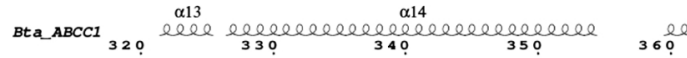

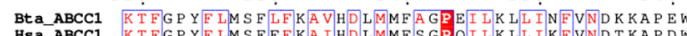

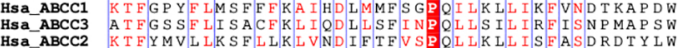

Hsa_ABCC

Sf_-ABCC2

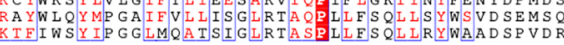

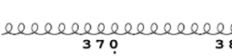

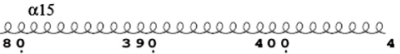

$\alpha 16$

LT R A I I I
I W K A M R
E T K K V V L F

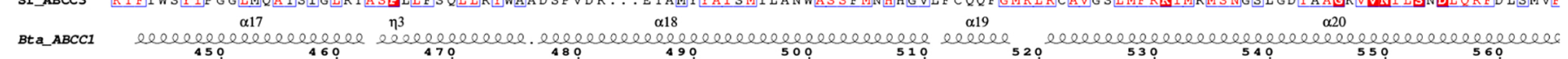

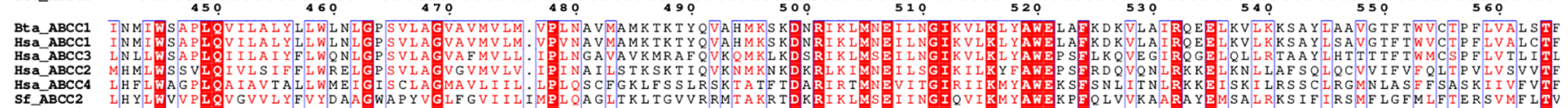

Sf_ABCC

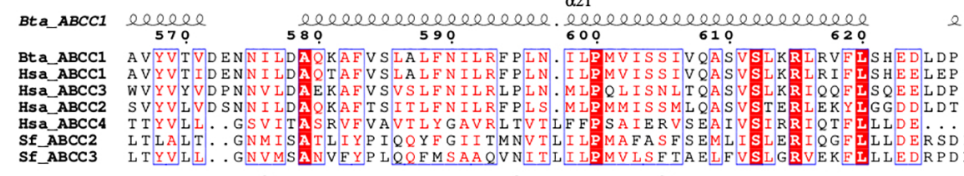

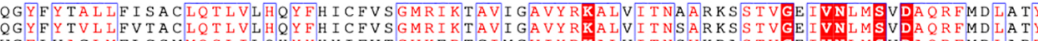

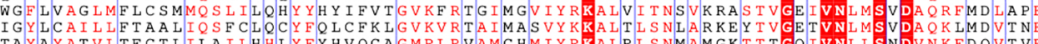

Bta_ABCCI

Bta_ABCC1

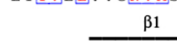

640 $\frac{\beta 1}{650}$
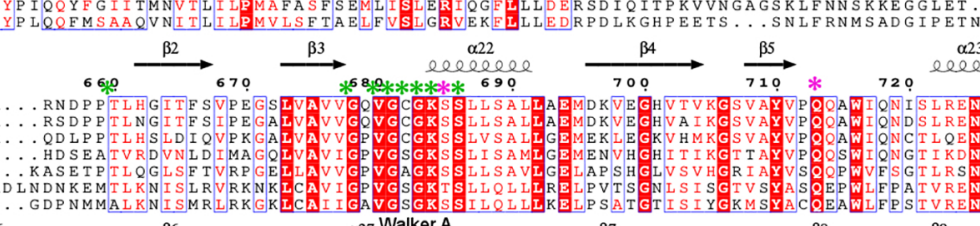
c23

630

DSIRRRPIKD

QSVERKTIS
SAIRHDCNFD

IS SR RRQLP
IIIARMQDEP

Hsa_ABCC

Hsa_ABCCA
Sf_ABCC
Sf_ABCC

YAITIHSGTFT TAM
KAMOFSEAST

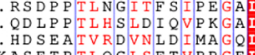

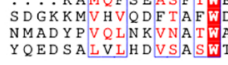

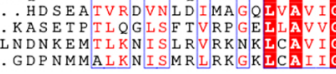

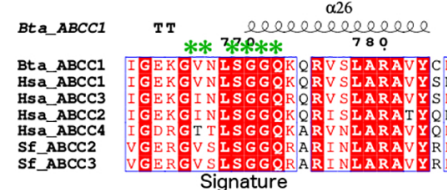

B6 $\quad 027$ Walker $A$
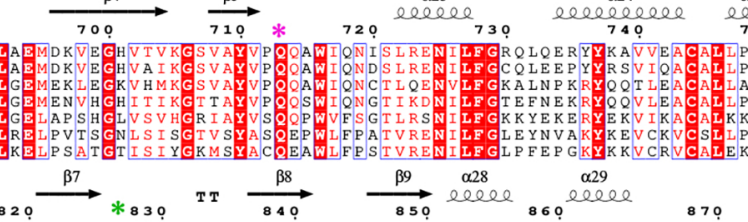

o25
750 ?

VEKETRSVS

\section{Bta_ABCCI}

Bta_ABCC1 GVGGP. GKEVK QMENGM.

Hsa_ABCC1 GVSGP.GKEARQMENGM
Hsa_ABCC3 ALEGAEDKEALIIEDTI

$\begin{array}{ll}\text { Hsa_ABCC3 } & \text { ALEGGEDKEALIIEDTI } \\ \text { Hsa_ABCC2 } & \text { EEDDDYGLISSVEEIPE }\end{array}$

Hsa_ABCC4
Sf_ABCC2

S1_ABCC
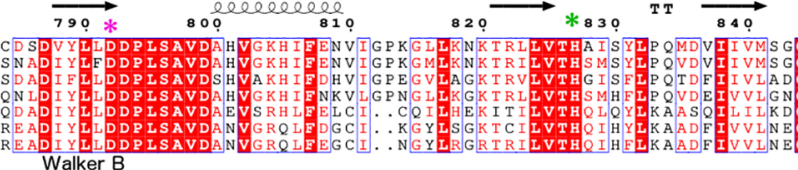

Walker B

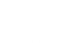

920
$92.5 \mathrm{~T}$.

$$
930
$$

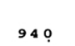

VVLNE

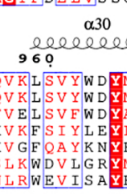

o.290e

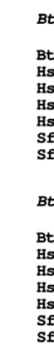

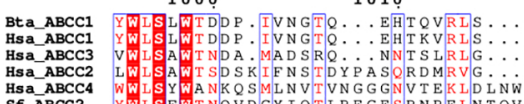

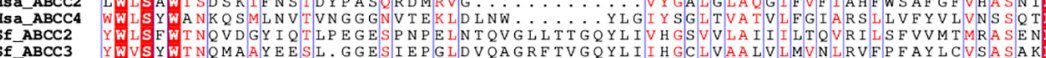

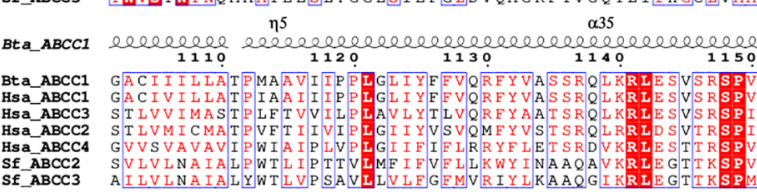

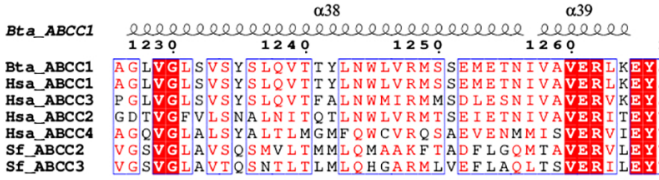

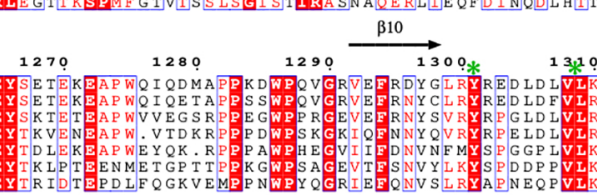
$\underset{116}{\rightarrow} \alpha 42$

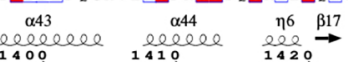

Bta_ABCC

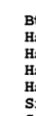

\section{Hsa}
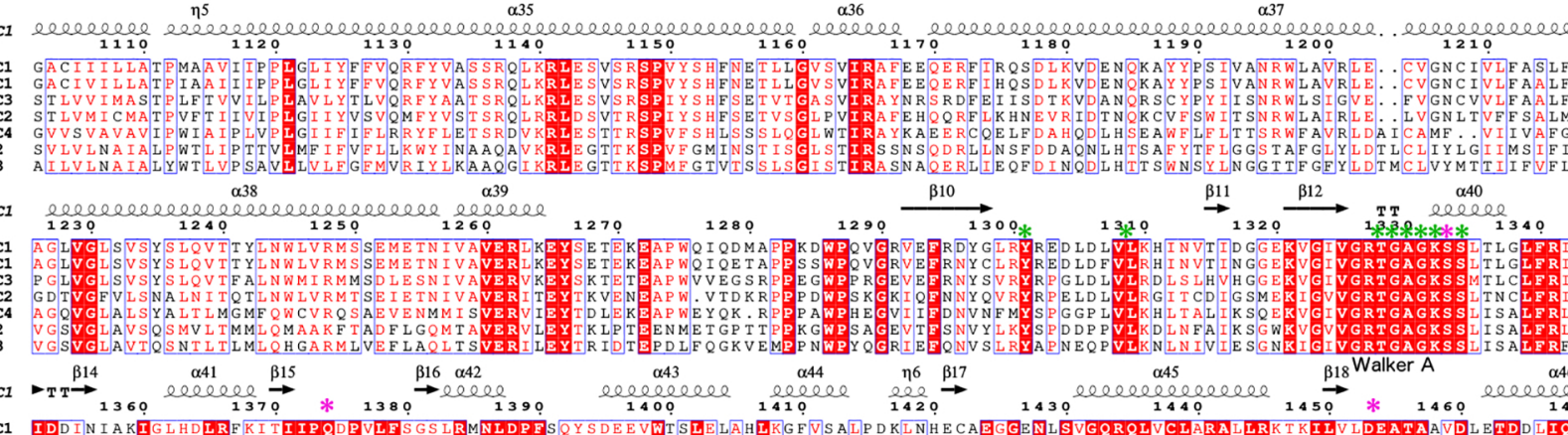

1210 . 1220

$\alpha 31$

30

980 cerereen

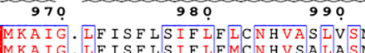

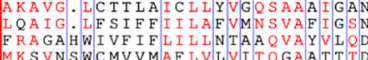

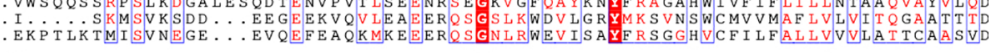

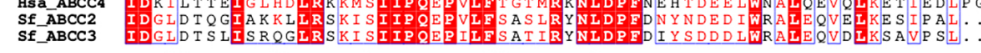
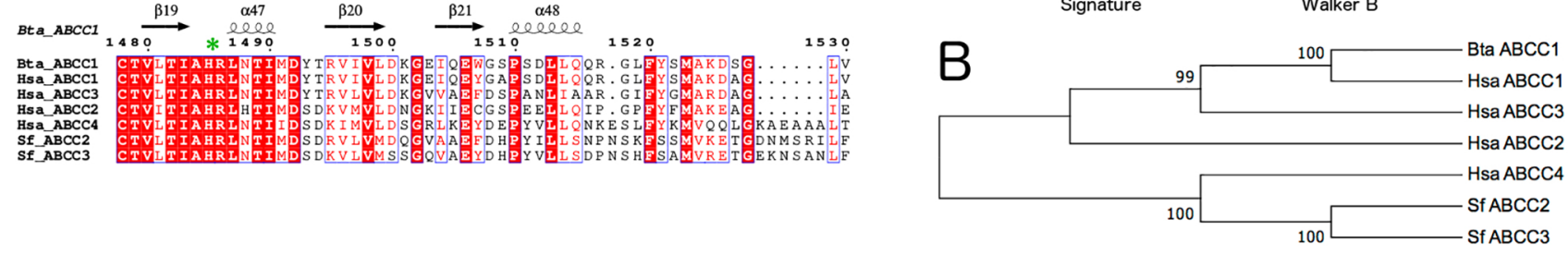


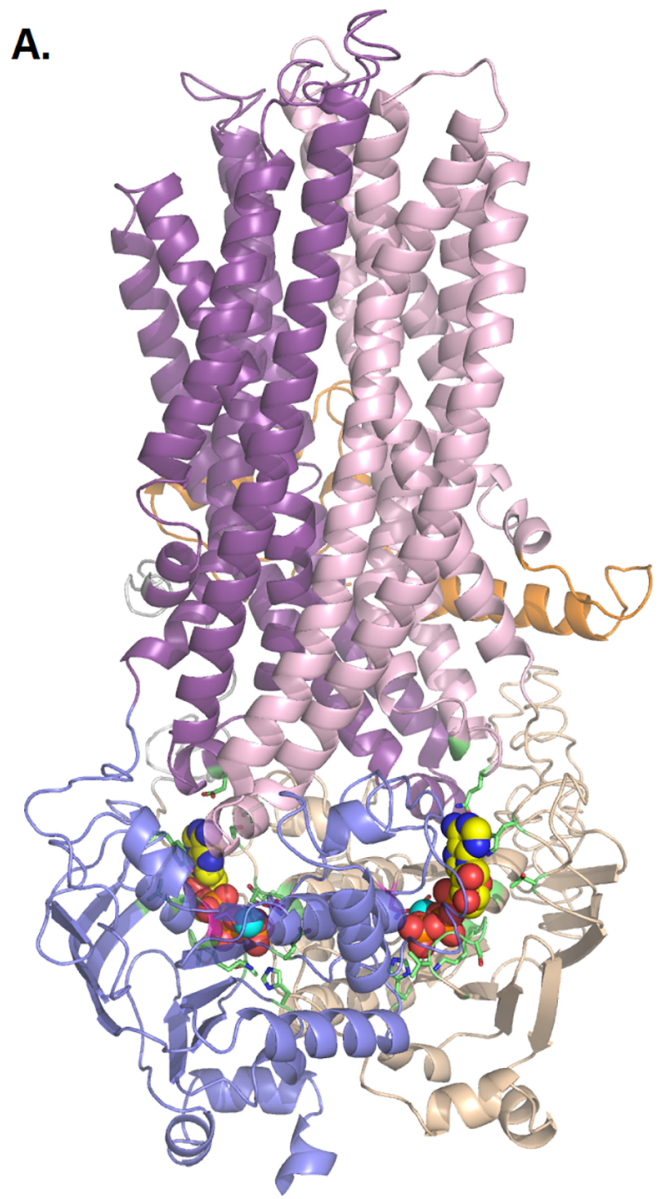

B.
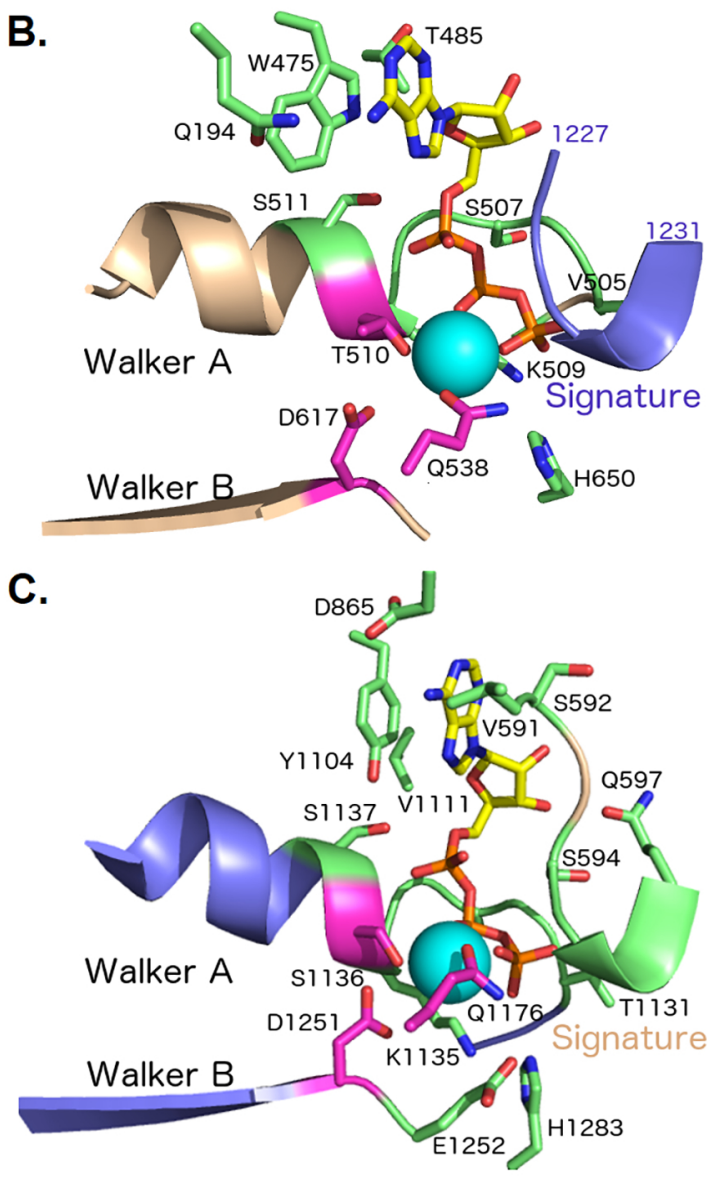

Figure 5 